\title{
All trans-retinoic acid (ATRA) induces re-differentiation of early transformed breast epithelial cells
}

\author{
MARIA F. ARISI ${ }^{1}$, REBECCA A. STARKER ${ }^{1}$, SANKAR ADDYA $^{1}$, YONG HUANG ${ }^{2}$ and SANDRA V. FERNANDEZ ${ }^{1}$ \\ ${ }^{1}$ Kimmel Cancer Center, Thomas Jefferson University, Philadelphia, PA 19107; ${ }^{2}$ Section of Gastroenterology, \\ Department of Medicine, University of Chicago, Chicago, IL 60637, USA
}

Received December 23, 2013; Accepted February 3, 2014

DOI: $10.3892 /$ ijo.2014.2354

\begin{abstract}
Retinoids have been used as potential chemotherapeutic or chemopreventive agents because of their differentiative, anti-proliferative, pro-apoptotic and antioxidant properties. We investigated the effect of all trans-retinoic acid (ATRA) at different stages of the neoplastic transformation using an in vitro model of breast cancer progression. This model was previously developed by treating the MCF-10F human normal breast epithelial cells with high dose of estradiol and consists of four cell lines which show a progressive neoplastic transformation: MCF-10F, normal stage; trMCF, transformed MCF-10F; bsMCF, invasive stage; and caMCF, tumorigenic stage. In 3D cultures, MCF-10F cells form tubules resembling the structures in the normal mammary gland. After treatment with estradiol, these cells formed tubules and spherical masses which are indicative of transformation. Cells that only formed spherical masses in collagen were isolated (trMCF clone 11) and treated with ATRA. After treatment with 10 or $1 \mu \mathrm{M}$ ATRA, the trMCF clone 11 cells showed tubules in collagen; 10 and $43 \%$ of the structures were tubules in cells treated with 10 and $1 \mu \mathrm{M}$ ATRA, respectively. Gene expression studies showed that 207 genes upregulated in transformed trMCF clone 11 cells were downregulated after $1 \mu \mathrm{M}$ ATRA treatment to levels comparable to those found in the normal breast epithelial cells MCF-10F. Furthermore, 236 genes that were downregulated in trMCF clone 11 were upregulated after $1 \mu \mathrm{M}$ ATRA treatment to similar levels shown in normal epithelial cells. These 443 genes defined a signature of the ATRA re-programming effect. Our results showed that $1 \mu \mathrm{M}$ ATRA was able to re-differentiate transformed cells at early stages of the neoplastic process and antagonistically regulate breast cancer associated genes. The
\end{abstract}

Correspondence to: Dr Sandra V. Fernandez, Medical Oncology Department, Kimmel Cancer Center, Thomas Jefferson University, 233 South 10th Street, 1002 BLSB, Philadelphia, PA 19107, USA

E-mail: sandra.fernandez@jefferson.edu

Key words: breast cancer, vitamin A, retinoic acid, transformation, branching invasive and tumorigenic cells did not show any changes in morphology after ATRA treatment. These results suggest that ATRA could be used as a chemopreventive agent to inhibit the progression of premalignant lesions of the breast.

\section{Introduction}

Vitamin A is obtained through the diet in the form of retinol, retinyl ester or $\beta$-carotene (1). Retinoic acid (RA) is one of the principal active metabolites of vitamin A which plays a critical role in cell proliferation, differentiation and apoptosis in normal tissues during embryonic development (2). RA induces differentiation in many cell types and is the most widely used differentiating therapeutic agent $(3,4)$. Retinol has 6 biologically active isoforms that among others includes all-trans (ATRA, tretinoin) and 9-cis RA (alitretinoin); ATRA is the predominant physiological form (5). RA mediates the transcriptional regulation of several genes by binding to the nuclear retinoic acid receptors (RARs), namely RAR $\alpha$, RAR $\beta$ and RAR $\gamma(6,7)$. Like other nuclear receptors, RARs contain a domain that mediates interaction with ATRA, a zinc finger-containing DNA binding domain that binds to RA response elements (RAREs) in target genes, and a dimerization domain that engages members of the retinoid $\mathrm{X}$ receptor (RXR) subfamily in RXR/RAR heterodimers (8). Different isomers activate different receptors and thus lead to different biological effects. RARs can be activated by both all-trans (ATRA) and 9-cis-RA, while RXR are exclusively activated by 9-cis RA; however, due to the conversion of ATRA to 9-cis RA, high concentrations $\left(10^{-5} \mathrm{M}\right)$ of ATRA can also activate gene transcription in cells transfected with RXRs (9). It has also been shown that retinoids exert their effects via the nuclear receptor independent pathway (5).

RA and its derivatives are promising anti-neoplastic agents endowed with both therapeutic and chemo-preventive potential because they are able to regulate cell growth, differentiation and apoptosis $(10,11)$. It is believed that the anti-neoplastic pathways induced by RA are regulated predominantly by RAR- $\beta$, which is known to induce apoptosis; thus it has been suggested that $\operatorname{RAR}-\beta$ plays a critical role in mediating the growth arrest and differentiation in several breast cancer cell types (12-14).

We have developed an in vitro-in vivo model of breast cancer progression by treating the human normal-like breast 


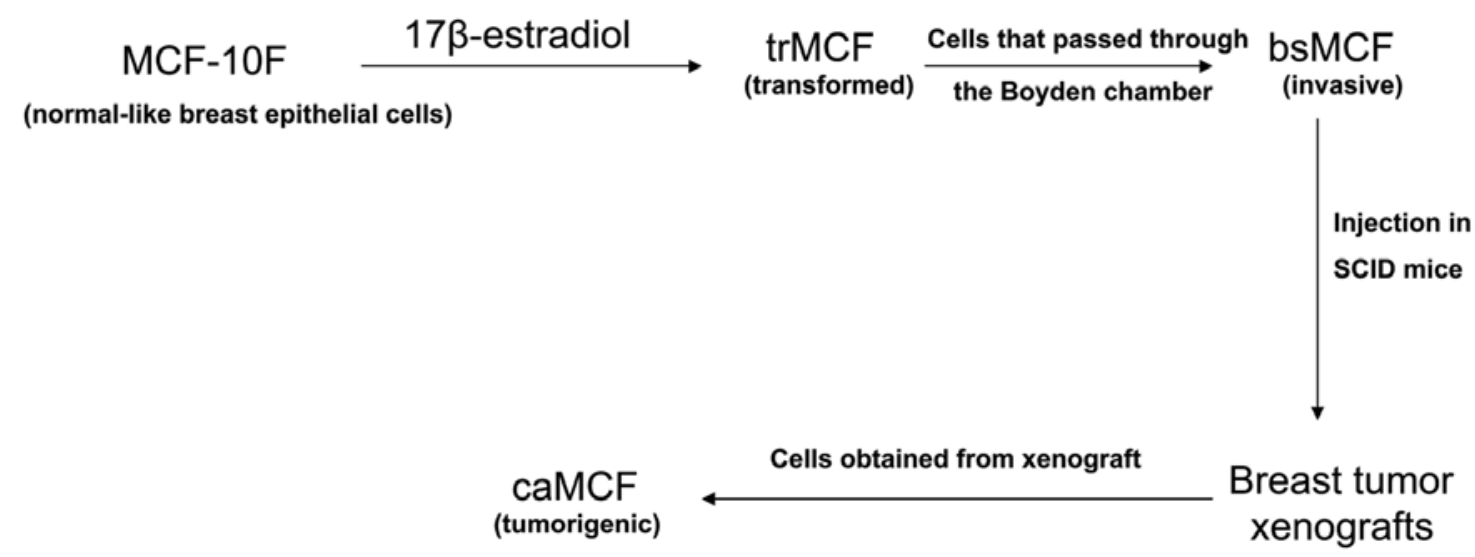

Figure 1. In vitro-in vivo model of cell transformation. The human normal-like MCF-10F cells were treated with high dose of estradiol and named early transformed breast epithelial cells (trMCF). The trMCF cells were seeded on a Boyden chamber and the cells that invaded, bsMCF, were selected and expanded. The bsMCF cells were injected in the fat mammary pad of SCID mice producing breast tumor xenografts. These xenografts were surgically removed and tumor cells were expanded giving origin to caMCF. The trMCF cells did not produced tumors when injected in SCID mice.

epithelial cells MCF-10F with a high dose of estradiol (70 nM) (Fig. 1) $(15,16)$. This model consists of four cell lines: i) the spontaneously immortalized cell line MCF-10F, which is considered to be a normal-like breast epithelial cell line; ii) the transformed trMCF cells; iii) the invasive bsMCF cells; and iv) cells isolated from xenografts, caMCFs, which show all characteristics of fully malignant breast cancer cells (Fig. 1). Gene expression studies showed the highest number of deregulated genes in caMCF, being slightly lower in bsMCF, and lowest in trMCF and, this order was consistent with the extent of chromosome aberrations (caMCF $>$ bsMCF $>>$ trMCF) (16). This model of breast cancer progression resembles the different steps of neoplastic transformation of the mammary gland; it is widely held that breast cancer initiates as the premalignant stage of atypical ductal hyperplasia (ADH), progresses into the pre-invasive stage of ductal carcinoma in situ (DCIS), and culminates in the potentially lethal stage of invasive ductal carcinoma (IDC) (17). In collagen, the normal-like MCF-10F cells form tubules resembling the structures observed in the normal mammary gland although after treatment with estradiol, the transformed trMCF cells form tubules and spherical masses, which are indicative of cell transformation $(8,19)$. The spherical masses showed a partial filling of the lumen that would result from decreased central apoptosis, enhanced cellular proliferation or both (18). The filling of the lumen of the tubular structures of the breast is the earliest morphologic alteration and is common in atypical ductal hyperplasia and ductal carcinoma in situ (DCIS) (18). In the presented study, we studied the effect of all trans-RA (ATRA) using this model of breast cancer progression. Our results showed that ATRA was able to re-program early transformed cells to a normal stage.

\section{Materials and methods}

Cells and media. The human normal-like breast epithelial cells MCF-10F are estrogen receptor (ER) negative, progesterone receptor (PR) negative and HER2 negative. Cells were cultured in Dulbecco's modified Eagle's medium [DMEM/F-12, Gibco, Carlsbad, CA; formula 90-5212 EF: containing DMEM/F12 (1:1) with L-glutamine and phenol red, D-glucose $315 \mathrm{mg} / \mathrm{l}$, sodium pyruvate $55 \mathrm{mg} / \mathrm{l}$ ] with $5 \%$ horse serum, $2.43 \mathrm{~g} / \mathrm{l}$ sodium bicarbonate, $20 \mathrm{mg} / 1$ epidermal growth factor (EGF), $100 \mathrm{mg} / \mathrm{l}$ Vibrio cholerae toxin, $10 \mathrm{mg} / \mathrm{l}$ insulin, $0.5 \mathrm{mg} / 1$ hydrocortisone, $1.05 \mathrm{mM}$ calcium, antibiotics and antimicotic $(100 \mathrm{U} / \mathrm{ml}$ penicillin, $100 \mathrm{mg} / \mathrm{ml}$ streptomycin, $0.25 \mathrm{mg} / \mathrm{ml}$ amphotericin). A 10-mM solution of all trans-retinoic acid (ATRA, Cat\# R2625, Sigma, St. Louis, MO) was prepared as a stock solution by dissolving ATRA in dimethylsulphoxide (DMSO).

The trMCF clone 11 was isolated by seeding $100-1,000$ trMCF cells in a 100-mm cell culture plate and after 1 day in culture, several colonies were isolated by ring cloning. The trMCF clone 11 cells were generated by expanding the cells from one of these colonies; trMCF clone 11 cells only formed spherical masses on collagen. To study the effect of ATRA, trMCF clone 11 cells were treated continuously for 26 days with $10^{-5} \mathrm{M}(10 \mu \mathrm{M})$ to $10^{-8} \mathrm{M}(0.01 \mu \mathrm{M})$ ATRA (media was replaced daily). As control, the cells were treated with $0.1 \%$ DMSO (vehicle). The bsMCF and caMCF cells were treated with $10^{-5}$ to $10^{-8} \mathrm{M}$ ATRA alone or in combination with $2.5 \mu \mathrm{M}$ 5-aza-dC.

Collagen assays. The cells were resuspended at a final density of $1.5 \times 10^{4}$ cells $/ \mathrm{ml}$ in collagen matrix consisting of $2.68 \mathrm{mg} / \mathrm{ml}(89.3 \%)$ type I collagen (PureCol, Inamed Biomaterials Co., Fremont, CA), 8\% 12.5X DMEM-F12 with antibiotics, $0.1 \mathrm{mg} / \mathrm{ml}$ insulin, $14 \mathrm{mM} \mathrm{NaHCO}$ and $0.01 \mathrm{~N} \mathrm{NaOH}$. A total of $400 \mu 1$ (3,000 cells) were plated on the top four 24-well chambers pre-coated with $400 \mu \mathrm{l}$ of $89.3 \%$ collagen mix. Per each treatment, cells were plated in 4 wells and fed daily with the medium described before. The structures in collagen matrix were observed daily under an inverted microscope and at the end of the observation period (8 days), the structures (spherical masses, tubules and intermediate structures) were counted, photographed and fixed in $10 \%$ neutral buffered formalin and processed for histological examination. Results were expressed as the total number of structures per well (spherical masses, tubules and intermediate structures) and percentage of the different structures per treatment. The t-test was used to determine if the differences were significant. 

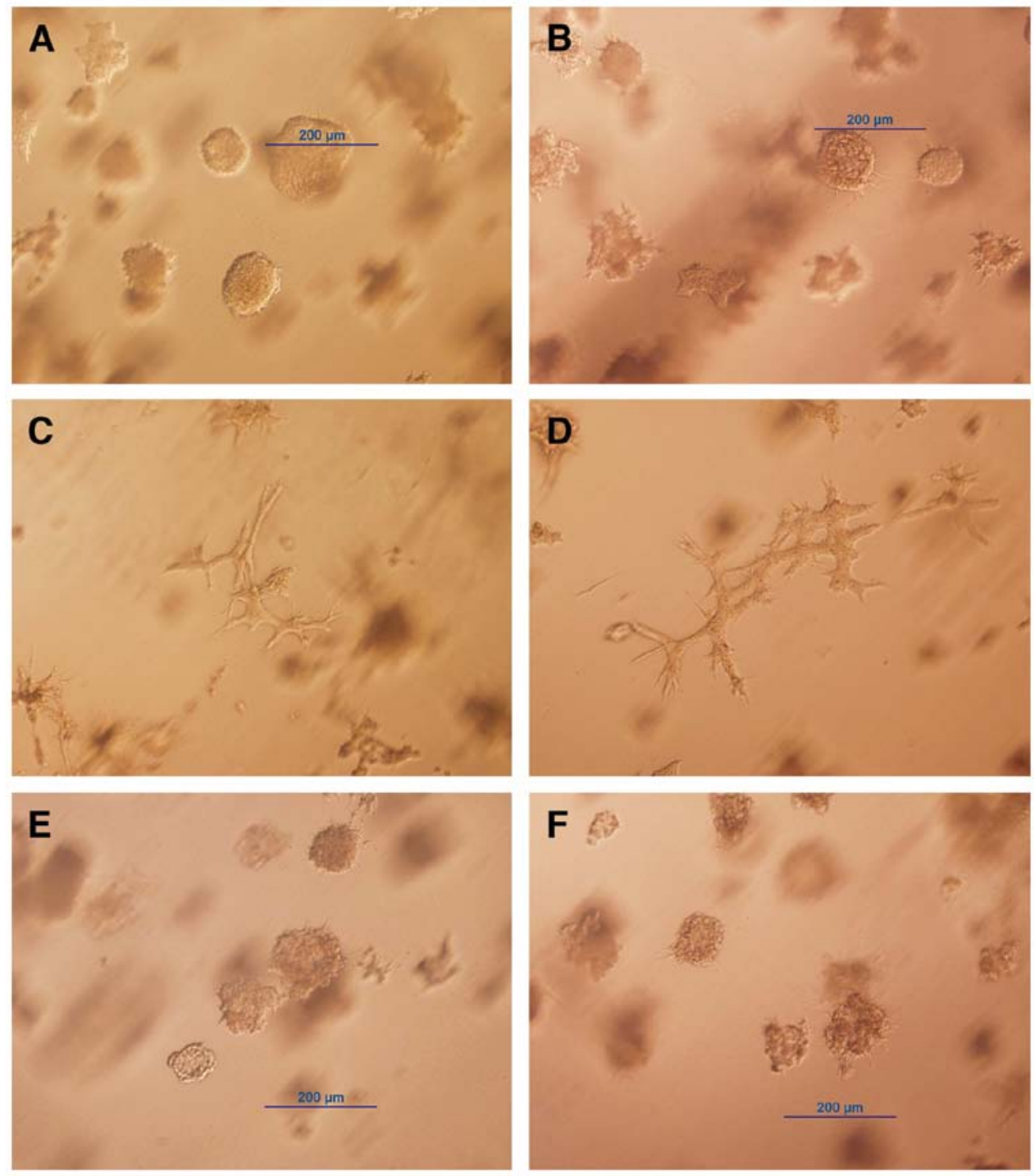

Figure 2. All-trans retinoic acid (ATRA) induces morphological changes in transformed cells trMCF clone 11. The trMCF clone 11 cells were plated in collagen matrix (3-D cultures) after being treated continuously for 26 days with different concentrations of ATRA. The cells were photograph after 8 days in collagen. (A) trMCF clone 11 cells (control); (B) trMCF clone 11 cells treated with $0.1 \%$ DMSO (vehicle, control); (C) trMCF clone 11 after being treated with $10^{-5} \mathrm{M}(10 \mu \mathrm{M})$ ATRA; (D) trMCF clone 11 cells after being treated with $10^{-6} \mathrm{M}(1 \mu \mathrm{M})$ ATRA; (E) trMCF clone 11 cells after being treated with $10^{-7} \mathrm{M}$ ATRA; (F) trMCF clone 11 cells after being treated with $10^{-8} \mathrm{M}$ ATRA.

Invasion assays. Cell invasion in real-time were performed using xCELLigence RTCA DP device from Roche Diagnostics (Mannheim, Germany). For this purpose, each well of the upper chamber of the CIM-Plate 16 was covered with Matrigel (BD Biosciences, Franklin Lakes, NJ) basement membrane matrix (1:20 in cell culture media) and 10\% fetal bovine serum (chemo-attractant) was added in the lower chamber. A total of 40,000 cells suspended in $100 \mu \mathrm{l}$ serum free media were seeded per well in CIM-Plates 16 (Roche Diagnostics). Data acquisition and analysis was performed with the RTCA software (version 1.2, Roche Diagnostics). Changes in impedance from cells that invade and migrate to the underside of wells were recorded and monitored for a total of $24 \mathrm{~h}$.

Gene expression profiling. RNA was isolated from the cells using RiboPure ${ }^{\mathrm{TM}}$ kit (Life Technologies, Frederick, MD) and RNA quality was controlled using the Agilent 2100
Bioanalyzer. Gene expression studies were performed using Affymetrix U133 Plus 2.0 (Affymetrix, Santa Clara, CA) human oligonucleotide microarrays containing over 47,000 transcripts and variants, including 38,500 well characterized human genes. After hybridization, the chips were scanned using GeneChip Scanner 3000. The data were analyzed with Microarray Suite version 5.0 (MAS 5.0) using Affymetrix default analysis settings and global scaling as normalization method. The trimmed mean target intensity of each array was arbitrarily set to 100 . Background correction and normalization was done using Iterative Plier 16 with GeneSpring V11.5 software (Agilent, Palo Alto, CA). The criteria for differentially expressed genes was set at $\geq 2$-fold changes ( $p$-value $<0.05$ ). The differentially expressed gene list was loaded into Ingenuity Pathway Analysis (IPA) 8.0 software (Ingenuity Systems, Redwood City, CA) to perform biological network and functional analyses. 
A

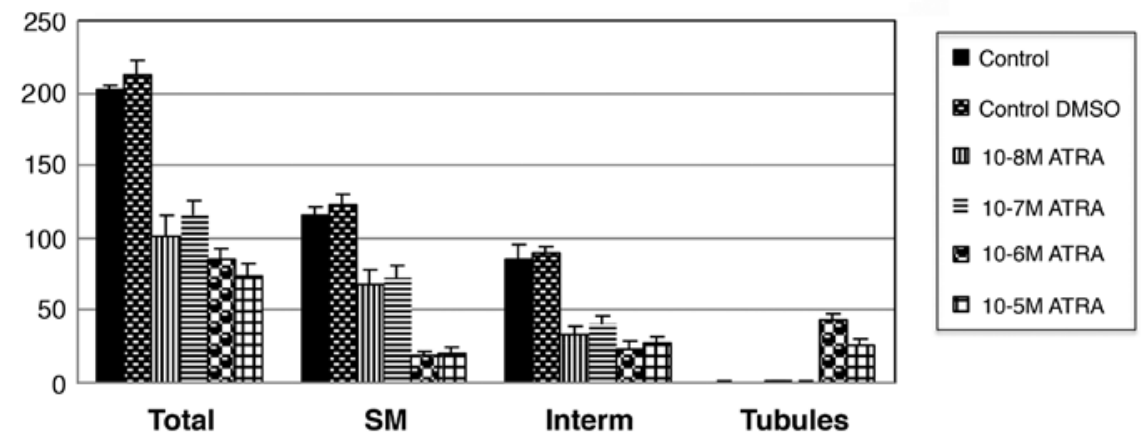

B

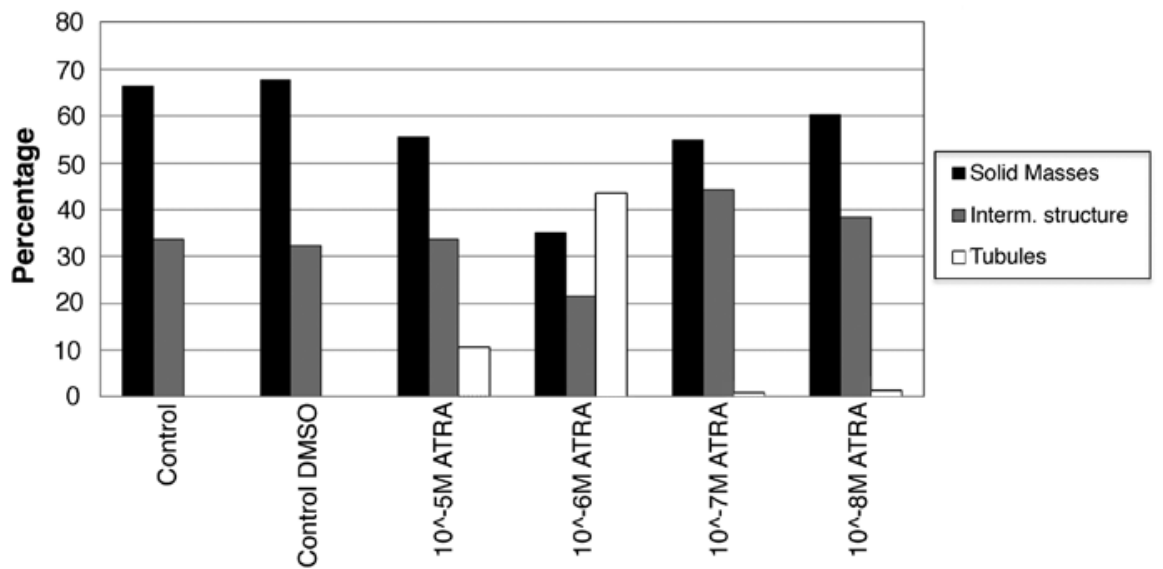

Figure 3. Spherical masses, tubules and intermediate structures formed in collagen by trMCF clone 11 before and after ATRA treatments. The trMCF clone 11 cells form spherical masses in collagen and some intermediate structures. The trMCF clone 11 cells were treated with different concentrations of all-trans retinoic acid (ATRA) for 26 days; after ATRA treatments, the cells were plated in collagen. (A) Total number of different structures in collagen of trMCF clone 11 cells before and after ATRA treatment. Total number of spherical masses (SM), tubules and intermediate structures (spherical masses with prolongations) per well are shown. (B) Different structures on collagen matrix of trMCF clone 11 cells after treatment with different concetrations of ATRA. Percentage of different structures in collagen.

\section{Results}

Treatment with ATRA induced branching morphogenesis in early transformed breast epithelial cells. MCF-10F cells are normal-like breast epithelial cells that form tubules in collagen matrix (3D culture); when these cells were treated with high dose of estradiol $(70 \mathrm{nM})$, the cells (trMCF) formed tubules and spherical masses. To isolate transformed cells that only form spherical masses, trMCF cells were seeded at low density in cell culture dishes and several clones were isolated by ring cloning. One of these clones, trMCF clone 11, did not form tubules in collagen; instead these cells formed spherical masses and intermediate structures (Fig. 2A and B). The trMCF clone 11 cells were treated continuously for 26 days with $10^{-5}$ to $10^{-8} \mathrm{M}$ all trans-retinoic acid (ATRA) and, we found that cells treated with $10^{-5}$ and $10^{-6} \mathrm{M}$ ATRA were able to form tubules in collagen (Fig. 2C and D). Furthermore, the spherical masses formed by trMCF clone 11 treated with $10^{-5}$ and $10^{-6} \mathrm{M}$ ATRA (Fig. 2C and D) were smaller compared to the ones formed by the controls (Fig. 2A and B) or cells treated with $10^{-7}$ and $10^{-8} \mathrm{M}$ ATRA
(Fig. 2E and F). The trMCF clone 11 cells treated with $10^{-7}$ and $10^{-8} \mathrm{M}$ ATRA (Fig. 2E and F) did not show any difference in morphology when compared to the controls (Fig. 2A and B). The number of spherical masses, intermediate structures and tubules for trMCF clone 11 cells treated with different concentrations of ATRA was counted (Fig. 3). The total number of structures in collagen was significantly lower in cells treated with ATRA compared with the controls suggesting that ATRA treatment decrease the proliferation rate of the cells $(\mathrm{p}<0.01)(\mathrm{Fig} .3 \mathrm{~A})$. The control trMCF clone 11 showed spherical masses and intermediate structures but no tubules in collagen while cells treated with $10^{-6}$ and $10^{-5} \mathrm{M}$ ATRA formed tubules and less spherical masses (Fig. 3A). The cells treated with $10^{-5}$ or $10^{-6} \mathrm{M}$ ATRA formed significantly less spherical masses than the cells treated with $10^{-7}$ or $10^{-8} \mathrm{M}$ ATRA ( $<<0.01$ ) (Fig. 3A). A total of $43 \%$ of the structures were tubules in the wells containing cells treated with $10^{-6} \mathrm{M}$ ATRA and $10 \%$ tubules in wells with $10^{-5}$ M ATRA-treated cells (Fig. 3B).

The invasion capacity of trMCF clone 11 was studied before and after ATRA treatment but, no differences were 
trMCF clone 11

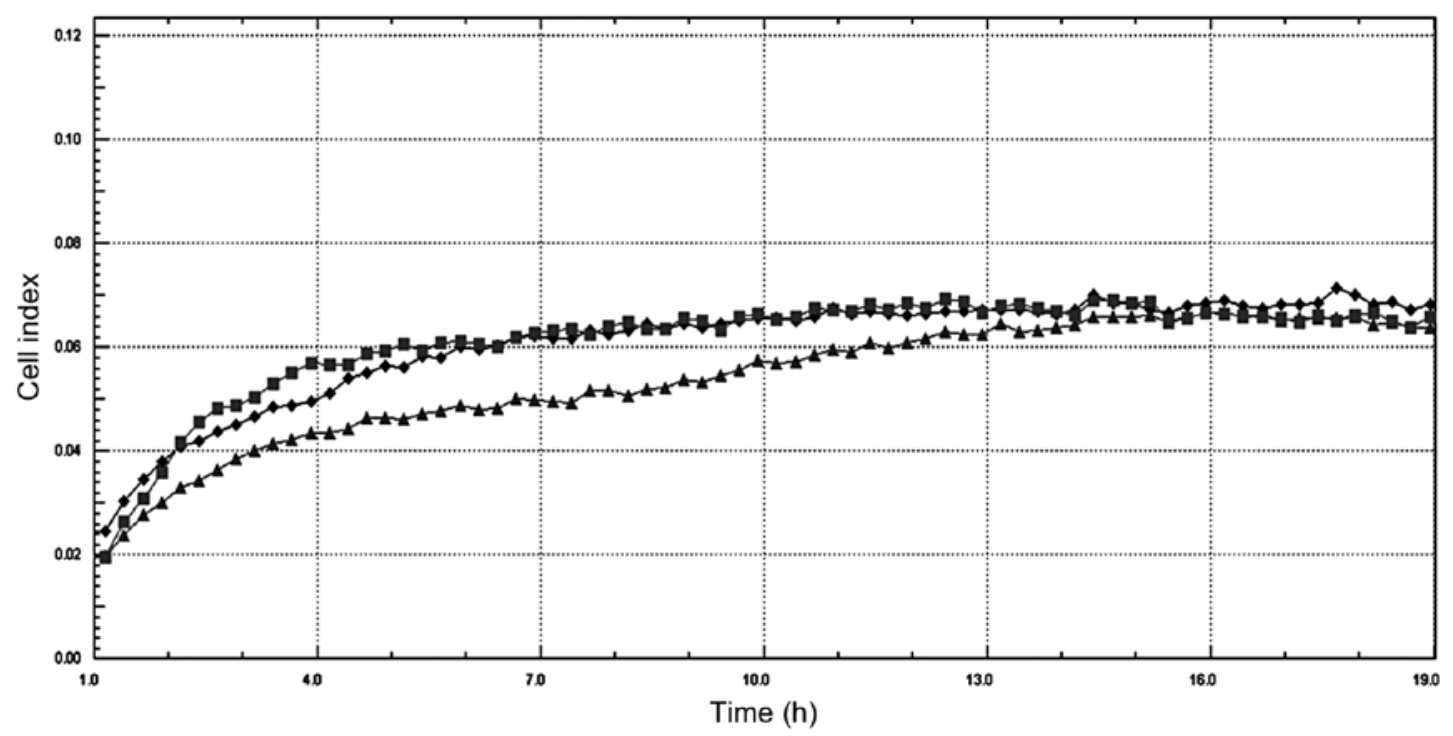

Figure 4. Invasion assay of trMCF clone 11 before and after ATRA treatments. The cell index of Matrigel-coated wells (invasion) at different time points are shown. The invasion capacity of the trMCF clone 11 did not show significant differences after $10^{-5} \mathrm{M}$ ATRA ( $\left.\mathbf{(}\right)$ or $10^{-6} \mathrm{M}$ ATRA (๘) compared with control after $16 \mathrm{~h}$.

observed (Fig. 4). The bsMCF and caMCF cells did not show any changes in their morphology or invasion capacity after treatment with ATRA alone or in combination with the demethylating agent 5-aza-cytidine (data not shown).

Treatment with ATRA re-programmed gene expression of early transformed cells. As trMCF clone 11 cells that only formed spherical masses on collagen were able to form tubules after treatment with $10^{-5}$ or $10^{-6} \mathrm{M}$ ATRA, gene expression studies were performed on these cells. The microarray data have been deposited into the NCBI gene expression omnibus (GEO) datasets (GSE51549). The unsupervised sample classification by PCoA (principle coordinate analysis) revealed that trMCF clone 11 cells treated with $10^{-5}$ or $10^{-6} \mathrm{M}$ ATRA demonstrated a major difference with trMCF clone 11 cells, and a minor difference with MCF-10F; also sample differences between $10^{-5} \mathrm{M}$ ATRA and $10^{-6} \mathrm{M}$ ATRA were weak (Fig. 5). Although, trMCF clone 11 cells treated with $10^{-5} \mathrm{M}$ ATRA and $10^{-6} \mathrm{M}$ ATRA showed minor differences at the expression level, we considered trMCF clone 11 treated with $10^{-6} \mathrm{M}$ ATRA for the expression analysis since the number of tubules in collagen matrix was higher for this concentration (43\% tubules with $10^{-6} \mathrm{M}$ ATRA vs. $10 \%$ tubules with $10^{-5} \mathrm{M}$ ATRA). For gene expression studies, three experimental groups were compared using empirical Bayesian-moderated t-test implemented in R package 'limma': the normal breast epithelial cells MCF-10F, the cells transformed by treatment with estradiol trMCF clone 11 (that only formed spherical masses on collagen) and the trMCF clone 11 after treatment with $10^{-6} \mathrm{M}$ ATRA. We generated three gene lists at criteria of fold change $\geq 2$ and $\mathrm{p} \leq 0.05$ : gene list- 1 (trMCF clone11 vs. MCF-10F) with 1,409 probes (613 probes upregulated; 796 probes downregulated), gene list-2 (ATRA trMCF clone 11 vs. trMCF clone 11$)$ with 1,859 probe sets $(1,053$ probes upregulated; 806 probes downregulated) and gene list-3 (ATRA trMCF clone 11 vs. MCF-10F) with 870 probe sets (308 probes upregulated; 562 probes downregulated) (Fig. 6). Most importantly, 207 genes (271 probes) upregulated in the transformed trMCF clone 11 (compared to the normal MCF-10F) were downregulated after treatment with $10^{-6} \mathrm{M}$ ATRA (Fig. 6 and Table IA) and 236 genes (316 probes) that were downregulated in trMCF clone 11 (compared to MCF-10F) were upregulated by $10^{-6} \mathrm{M}$ ATRA treatment (Fig. 6 and Table IB). These 443 genes defined a gene signature programming the reverse-transformation effect by ATRA (Table I). The relatively smaller number of significant probe sets in gene list-3 compared with other gene lists (Fig. 6) further supported the findings that ATRA-treatment reprograms the gene expression status of trMCF clone 11 cells to MCF-10F.

Ingenuity pathway analysis (IPA) revealed 4 canonical pathways significantly dysregulated in the transformed cells trMCF clone 11: aryl hydrocarbon receptor signaling, retinoic acid activation, xenobiotic metabolism signaling and molecular mechanism of cancer (Table II). Several genes of these pathways that were up- or downregulated in trMCF clone 11 show similar levels of expression to MCF-10F after trMCF clone 11 was treated with $10^{-6} \mathrm{M}$ ATRA (Table II and Fig. 7). Genes from the aryl hydrocarbon receptor signaling ALDH1A3, CCND1, TGFBR2, TGM2 and TFDP1 were downregulated in the transformed cells trMCF clone 11 when compared to their expression in the normal breast epithelial cells MCF-10F and, the expression of these genes was upregulated after these cells were treated with $10^{-6} \mathrm{M}$ ATRA reaching similar levels to the expression in MCF-10F (Table II and Fig. 7). One of the functions that show enrichment of dysregulated genes in the transformed trMCF clone 11 cells is cell morphology and the expression of most of these genes reached similar levels to 


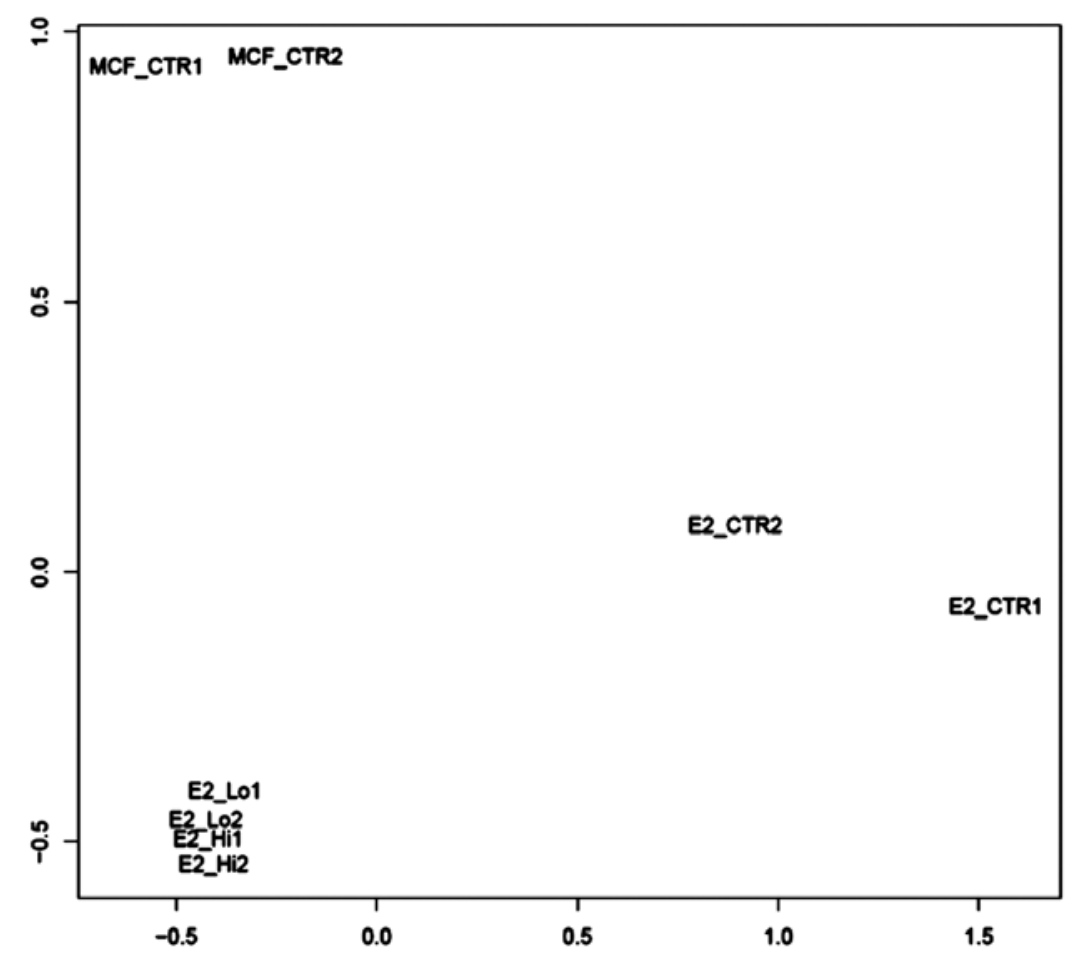

Figure 5. Unsupervised sample classification by principle coordinate analysis (PCoA). Two arrays were performed for each cell line and treatment: MCF-10F (MCF_CTR1 and MCF_CTR2), trMCF clone 11 (E2_CTR1 and E2_CTR2), $10^{-5} \mathrm{M}$ ATRA trMCF clone 11 (E2_Hi1 and E2_Hi2) and 10-6 M ATRA trMCF clone 11 (E2_Lo1 and E2_Lo2). The trMCF clone 11 cells treated with $10^{-5}$ M ATRA (E2_Hi) or 10 $0^{-6} \mathrm{M}$ ATRA (E2_Lo) shown a major difference with trMCF clone 11 cells (E2_CTR) and minor differences with MCF-10F cells (MCF_CTR).

Gene list-1

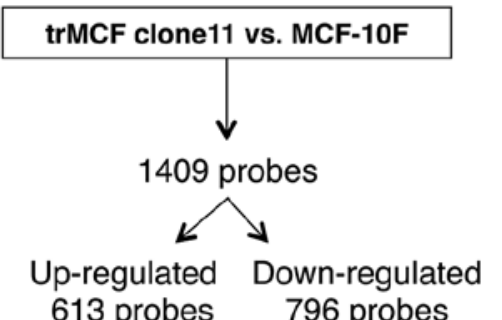

Gene list-2
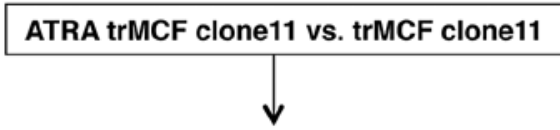

1859 probes

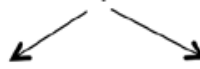

Up-regulated 1053 probes

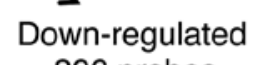

806 probes

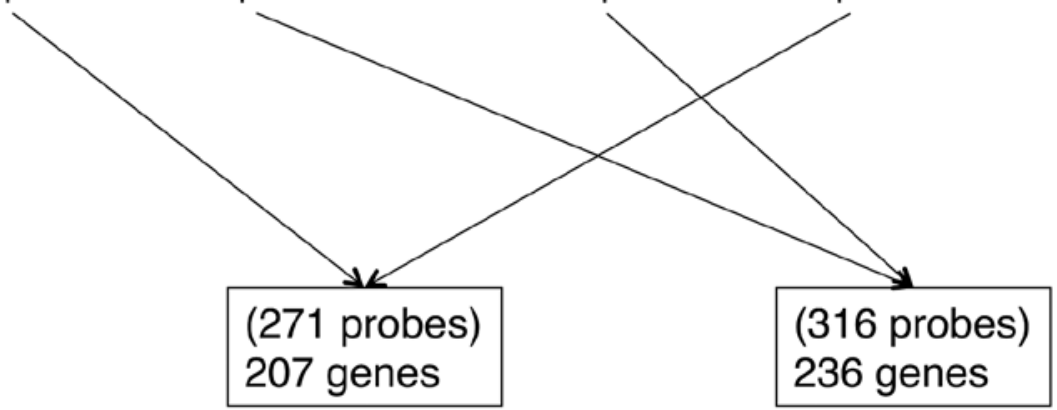

Gene list-3

ATRA trMCF clone11 vs. MCF

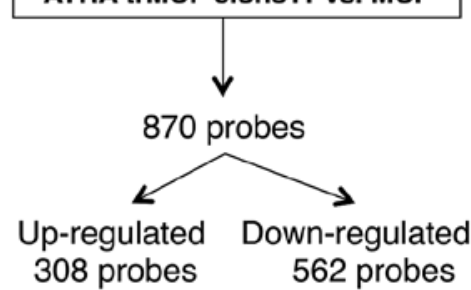

Figure 6. Representation of the gene expression studies showing number of dysregulated genes. Expression studies were performed in the early transformed trMCF clone 11 cells before and after treatment with $10^{-6}$ M ATRA (ATRA trMCF clone 11) and normal human breast epithelial MCF-10F cells. A total of 207 genes upregulated in the transformed trMCF clone 11 (compared to the normal MCF-10F) were downregulated after treatment with $10^{-6} \mathrm{M}$ ATRA and, 236 genes that were downregulated in trMCF clone 11 (compared to MCF-10F) were upregulated by $10^{-6} \mathrm{M}$ ATRA treatment. These 443 genes defined a gene signature programming the reverse-transformation effect by ATRA.

MCF-10F after trMCF cells were treated with $10^{-6} \mathrm{M}$ ATRA. The expression of some genes related to cell morphology such as PLD1, CD44, STX6, STMN3, ATF2, ETS2, NEK2,
HAS3, MGP, GNA13 were upregulated in the transformed trMCF clone 11 and their expression reached normal levels after $10^{-6} \mathrm{M}$ ATRA treatment (Fig. 7); other genes related to 
Table I. Gene signature programming.

A, ATRA-downregulated genes (207 genes).

\begin{tabular}{|c|c|c|c|c|}
\hline ACSS3 & DNAJB9 & KLF11 & PL-5283/SLC13A4 & TIMP3 $^{\mathrm{a}}$ \\
\hline ALDH3A2 & DSC3 & KLHDC8B & PLAG1 & TMEM167B \\
\hline ALDOC & DUSP5P & LCA5 & PLD1 & TMEM27 \\
\hline ALPK1 & EFHC1 & LOC100288092 & PLD6 & TMEM40 \\
\hline ANKRD37 & EFNB3 & LOC100289187 & PLK1S1 & TMEM59 \\
\hline AQPEP & EPB41L4B & LOC100505894 & POFUT2 & TNFRSF25 \\
\hline ARG2 & $\mathrm{ERCC}^{\mathrm{a}}$ & LOC100506057/STK32C & POLR1D & TNFSF11 \\
\hline ARHGAP19 & ETS2a & LOC100507303 & PPIL6 & TNKS \\
\hline ARHGEF10 & FABP6 & LOC100507547 & PPOX & TP63 \\
\hline ATF2 & FAM117A & LOC100507644 & PPP1R13L & TPD52L1 $^{\mathrm{a}}$ \\
\hline ATG14 & FAM168A & LOC439938 & PPP1R3C & TPRG1 \\
\hline $\mathrm{ATP} 2 \mathrm{C} 2$ & FAM46C & LOC642587 & PRKAB2 & TRAF3IP2 \\
\hline ATP5C1 & FAT2 & LOX & PRMT2 & TRAPPC6A \\
\hline BCAS4 & FBXO2 & LRIG1 & PROCR & TSC22D3 \\
\hline BFSP1 & FEM1B & LYST & PTEN & TTBK2 \\
\hline BLNK & FLCN & MAP2K5 & PTEN/PTENP1 & TTC39B \\
\hline BTBD3 & FLJ37644 & MAPT & PTPN14 & TXNIP \\
\hline C11orf80 & FLJ45244 & MGEA5 & RAB11FIP4 & UFM1 \\
\hline C16orf46 & FNBP1L & $\mathrm{MGP}^{\mathrm{a}}$ & RAB38 & UGT1A1/1A4/1A6/1A8/1A9/1A10 \\
\hline C17orf39 & FNTA & MLF1 & RAB40C & USP3 \\
\hline C17orf48 & FNTB & MRAP2 & RAB4A & USP32 \\
\hline C1orf133 & FSIP1 & MXD1 & RAB7L1 & VPS8 \\
\hline C1orf161 & FXYD2 & MYLIP & RASSF6 & WAC \\
\hline C20orf111 & GBAS & N4BP2L1 & RMND1 & WDR59 \\
\hline C21orf7 & GGNBP2 & NDE1 & RNF169 & WDR91 \\
\hline C5orf41 & GGTA1 & NDUFB4 & SCARA3 & WWOX ${ }^{\mathrm{a}}$ \\
\hline C7orf68 & GIT2 & NEK2 & SCRG1 & YOD1 \\
\hline C9orf9 & GJA3 & NEURL1B & SEMA6A & ZBTB34 \\
\hline CCDC28A & GKAP1 & NFKBIL1 & SFT2D1 & ZFAND5 \\
\hline CD44 ${ }^{\mathrm{a}}$ & GNA13 & NGLY1 & SHOX2 & ZNF836 \\
\hline CELSR2 & GNAI1 & NMNAT3 & SLC25A37 & ZNRF1 \\
\hline CLCA2 & GOSR2 & NPL & SLC2A12 & \\
\hline CMBL & GPM6A & OGFRL1 & SLC2A9 & \\
\hline COBL & GPNMB & PALMD & SLC5A3 & \\
\hline CRIP2 & H19 & $\mathrm{PDCD}^{\mathrm{a}}$ & SOCS3 & \\
\hline CSNK2A2 & HAS3 & PDCD5 & SORL1 & \\
\hline CYP1B1 & HBP1 & PDE7A & SPATA17 & \\
\hline CYP39A1 & HERPUD1 & PDZD2 & STAU2 & \\
\hline DBP & HMGCL & PER1 & STMN3 & \\
\hline DCD & IFNAR1 & PER3 & STX6 & \\
\hline DDAH2 & IRF6 & PHF21A & SUSD4 & \\
\hline $\mathrm{DDC}^{\mathrm{a}}$ & IRX2 & PHLDB3 & TESK2 & \\
\hline DDIT3 & $\mathrm{KCMF1}$ & PHTF2 & THBS2 & \\
\hline DHX40 & KDM5B & PIK3CD & THSD1///THSD1P1 & \\
\hline
\end{tabular}


Table I. Continued.

B, ATRA-upregulated genes (236 genes).

\begin{tabular}{|c|c|c|c|c|c|}
\hline ABHD13 & COX7B & HIATL1 & OSTM1 & SLC43A3 & TSPAN2 \\
\hline $\mathrm{ACP} 2$ & CRELD2 & HIGD1A & P2RY2 & SMPDL3A & $\mathrm{UBE}^{2} \mathrm{~N}^{\mathrm{a}}$ \\
\hline ADAM12 & CST6 $^{\mathrm{a}}$ & HOXA11 & PAPPA & SNRNP25 & UBE2Q1 \\
\hline ALDH1A3 & CSTF2 & HPGD & PARVA & SNX19 & UBP1 \\
\hline ANO1 & CYB561D2 & HS3ST1 & PCSK5 & SOAT1 & UNK \\
\hline AOX1 & DCBLD2 & HS6ST2 & PDE12 & SPAG1 & VARS2 \\
\hline APOL6 & DHRS9 & IFI44 & PHACTR3 & SPATA13 & VGLL3 \\
\hline ARGLU1 & DHX9 & IFIT3 & PHLDA1 & SRPX2 & VSIG10 \\
\hline ARHGAP26 & DNAJA1 & IFIT5 & PHLDA2 & SRSF10 & $\mathrm{ZADH} 2$ \\
\hline ARHGAP42 & DOLK & IFNAR1 & PITPNC1 & SRSF2IP & ZBED4 \\
\hline ARHGDIB & DPH3 & KHNYN & PKIB & SSPN & ZDHHC2 \\
\hline ARIH2 & EFCAB2 & KLHL18 & PLCXD2 & STK39 & ZMPSTE24 \\
\hline ASPHD2 & EFNB2 & KLHL23 & PLGLA/PLGLB1/PLGLB2 & STS & ZNF252 \\
\hline ATP6V0A2 & EHD4 & KRT80 & PNO1 & STYK1 & ZNF271 \\
\hline B3GALNT1 & EIF2AK1 & LOC100131993 & PNPLA3 & SUPT7L & ZNF326 \\
\hline BRI3BP & EIF5B & LOC100505759 & PODXL & SUSD5 & ZNF35 \\
\hline BTG1 & ELOVL6 & LOC100507192 & POLR3K & SYNCRIP & \\
\hline C12orf26 & $\mathrm{ENC}^{\mathrm{a}}$ & LOC283278 & PPP2R1B & SYNJ2BP & \\
\hline C12orf5 & ENY2 & LOC728903 & PRPS1 & SYTL2 & \\
\hline C1GALT1C1 & ERLIN2 & MACC1 & PRR15 & SYTL5 & \\
\hline C1orf116 & EXOG & MARCKS & PSCA & TBC1D30 & \\
\hline C1orf135 & FADS $1^{\mathrm{a}}$ & MAT2A & PSME3 & TFDP1 & \\
\hline C1orf212 & FAIM & MCFD2 & PTGR1 & TFRC & \\
\hline C1orf226 & FAM118B & MEIS3P1 & PTP4A2 & TGFB2 & \\
\hline C6orf223 & FAM119A & MFAP3L & PTPRB & TGFBR2 $2^{\mathrm{a}}$ & \\
\hline CALM1 ${ }^{\mathrm{a}}$ & FAM83A & MFI2 & PTPRJ & TGM2 & \\
\hline CCDC68 & FBXW2 & MFSD1 & RABIF & THSD4 & \\
\hline CCDC88A & FDX1 & MICALL1 & RBM25 & TIMM23 & \\
\hline $\mathrm{CCND}^{\mathrm{a}}$ & $\mathrm{FN} 1^{\mathrm{a}}$ & MMACHC & RBM45 & TIMM8A & \\
\hline $\mathrm{CDA}$ & FNIP2 & MRPL35 & RGS17 & TIMM8B & \\
\hline CDC42EP2 & FRMD3 & MST1R & RHOBTB1 & TLCD1 & \\
\hline $\mathrm{CDH} 2$ & FUCA1 & MTERFD3 & RHOF & TLR3 & \\
\hline CEP78 & FXN & MYEOV & RPL27A & TLR4 & \\
\hline CFH/CFHR1 & FZD8 & MYO5C & RPS6KA2 & TMC5 & \\
\hline CFI & GALNT7 & NAA40 & S1PR3 & TMEM133 & \\
\hline CHAC2 & GATAD2A & NAV3 & SAMHD1 & TMEM177 & \\
\hline CHML & GBP1 & NECAP1 & SCEL & TMEM9B & \\
\hline CHRNA5 & GDA & NIPAL1 & SGK223 & TP53I3 & \\
\hline CLDN23 & GGCX & NMI & SH3TC2 & TPCN2 & \\
\hline СМАН & GPATCH2 & NRP2 & SLC16A5 & TRAK2 & \\
\hline CNPY2 & GPX8 & NSD1 & SLC1A1 & TRIM45 & \\
\hline COL4A3 & GXYLT1 & OLAH & SLC35B4 & TRIOBP & \\
\hline COL4A4 & HAS2 & OR7E14P & SLC35C1 & TRNT1 & \\
\hline COX7A1 & HERC6 & OR7E47P & SLC37A1 & TSPAN12 & \\
\hline
\end{tabular}

Genes downregulated in trMCF clone 11 that were upregulated after $10^{-6} \mathrm{M}$ ATRA-treatment to similar levels found in the normal breast epithelial cells MCF-10F. 'aenes with binding sites for RAR $\alpha$ or RAR $\beta$ described by Hua et al (29). 
Table II. Canonical pathways enriched with differentially expressed genes.

trMCF clone 11 vs. MCF-10F

Aryl hydrocarbon

receptor signaling

\author{
ALDH1A3 2, CCND1 $\downarrow$, TGFBR2 $\downarrow$, TGM $2 \downarrow$, \\ TFDP1 $1, \mathrm{ALDH} 3 \mathrm{~A} 2 \uparrow, \mathrm{CYP} 1 \mathrm{~B} 1 \uparrow$
}

Other genes: CDKN1A $\downarrow, \mathrm{JUN} \downarrow$ ALDH7A1 $\uparrow$, $\mathrm{CSNK} 2 \mathrm{~A} 1 \uparrow, \mathrm{TGFB} 1 \uparrow, \mathrm{MAPK} 1 \uparrow, \mathrm{NFE} 2 \mathrm{~L} 2 \uparrow$

RAR activation

Xenobiotic metabolism signaling

Molecular mechanisms of cancer

\author{
ALDH1A3 $\downarrow$, DHRS9 $\downarrow$, NSD1 $\downarrow$, TGFB $2 \downarrow$, \\ CSNK $2 \mathrm{~A} 2 \uparrow, \mathrm{PIK} 3 \mathrm{CD} \uparrow, \mathrm{PRMT} 2 \uparrow, \mathrm{PTEN} \uparrow$ \\ Other genes: JUN $\downarrow, \mathrm{NR} 2 \mathrm{~F} 2 \downarrow, \mathrm{RBP} 1 \downarrow$, \\ CSNK2A $1 \uparrow$, CSNK2A $2 \uparrow$, MAPK $1 \uparrow$, \\ MAPK $14 \uparrow$, TGFB $1 \uparrow$, GNAS $\uparrow$
}

\author{
ALDH1A3 $\downarrow$, HS3ST1 $\downarrow$, HS6ST2 $\downarrow$, PPP2R1B $\downarrow$, \\ $\mathrm{ALDH} 3 \mathrm{~A} 2 \uparrow, \mathrm{CYP} 1 \mathrm{~B} 1 \uparrow, \mathrm{MAP} 2 \mathrm{~K} 5 \uparrow, \mathrm{PIK} 3 \mathrm{CD} \uparrow$, \\ UGT1A1 (and others UGT) \\ Other genes: CHST15 $\downarrow$, ALDH7A1 $\uparrow$, \\ CAMK1D $\uparrow$, HDAC4 $\uparrow$, MAPK $1 \uparrow$, MAPK $14 \uparrow$, \\ $\mathrm{MGMT} \uparrow, \mathrm{UGT} 8 \uparrow, \mathrm{NFE} 2 \mathrm{~L} 2 \uparrow$
}

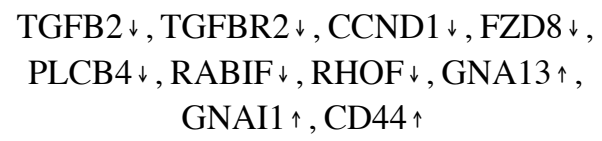

ATRA trMCF clone 11 vs. trMCF clone 11
$\mathrm{ALDH} 1 \mathrm{~A} 3 \uparrow, \mathrm{CCND} 1 \uparrow, \mathrm{TGFB} 2 \uparrow, \mathrm{TGM} 2 \uparrow$, TFDP1 1, ALDH3A2 2, CYP1B $1 \downarrow$

Other genes: CCNE $1 \uparrow, \mathrm{CCNE} 2 \uparrow, \mathrm{CDK} 6 \uparrow$, $\mathrm{DHFR} \uparrow, \mathrm{IL} 1 \mathrm{~B} \uparrow, \mathrm{IL} 6 \uparrow, \mathrm{NR} 2 \mathrm{~F} 1 \uparrow, \mathrm{NRIP} 1 \uparrow$, $\mathrm{POLA} 1 \uparrow \mathrm{ALDH} 3 \mathrm{~B} 2 \downarrow, \mathrm{ARNT} \downarrow, \mathrm{NCOA} 3 \downarrow$, HSPB $2 \downarrow$, ALDH6A1 $\downarrow$

ALDH1A3 $\uparrow$, DHRS9 $\uparrow$, NSD $1 \uparrow$, TGFB $2 \uparrow$, CSNK2A $2 \downarrow$, PIK3CD $\downarrow$, PRMT $2 \downarrow$, PTEN $\downarrow$

Other genes: GTF2H $2 \uparrow$, IGFBP $3 \uparrow$, MAP2K $1 \uparrow$, MAPK13 $\uparrow, N R 2 F 1 \uparrow, N R I P 1 \uparrow, \operatorname{RKAR} 2 B \uparrow$, DH10 1, CITED $2 \downarrow$, PNRC $1 \downarrow$, PRKAR1A $\downarrow$, SMARCD2 2

ALDH1A3, HS3ST1 1, HS6ST2 $\uparrow$, PPP2R1B $\uparrow$, ALDH3A $2 \downarrow$, CYP1B $1 \downarrow$, MAP2K5 $\downarrow$, PIK3CD $\downarrow$, UGT1A1 (and others UGT)

Other genes: ILIB $\uparrow$, IL6 $\uparrow$, NRIP $1 \uparrow$, MAP2K $1 \uparrow$, MAPK13 $\uparrow$, ALDH3B $2 \downarrow$, ARNT $\downarrow$, CAMK2D $\downarrow$, CITED2 $\downarrow$, MAP3K8 $\downarrow$, PPP2R3A $\downarrow$, ALDH6A1 $\downarrow$, MAP3K2 $\downarrow$

TGFB $2 \uparrow$, TGFBR $2 \uparrow$, CCND $1 \uparrow$, FZD $8 \uparrow$, PLCB $4 \uparrow$, RABIF $\uparrow$, RHOF $\uparrow$, GNA13 $\downarrow$, GNAI $1 \downarrow$, CD $44 \downarrow$

Other genes: $\mathrm{APC} \uparrow, \mathrm{CCNE} 1 \uparrow, \mathrm{CCNE} 2 \uparrow$, $\mathrm{CDC} 25 \mathrm{~A} \uparrow, \mathrm{CDK} 6 \uparrow, \mathrm{CYCS} \uparrow, \mathrm{E} 2 \mathrm{~F} 2 \uparrow$, MAP2K1 $\uparrow$, MAPK13 $\uparrow$, PRKAR2B $\uparrow$, RAPGEF3 $\uparrow$, RBL1 1, TFDP1 1, ARHGEF10 $\downarrow$, FOXO $1 \downarrow$, HHAT $\downarrow$, IRS $1 \downarrow$, NF $1 \downarrow$, PAK $3 \downarrow$, PIK3CD $\downarrow$, PRKAR1A $\downarrow$, RALGDS $\downarrow$, RHOV $\downarrow$

Genes downregulated $(\downarrow)$ or upregulated $(\uparrow)$ are shown. ATRA trMCF clone 11 refers to trMCF clone 11 treated with $10^{-6}$ M ATRA.

cell morphology such as PHLDA1, GBP1, HS6ST2 and TLR3 were downregulated in trMCF clone 11 and their expressions increased after $10^{-6} \mathrm{M}$ ATRA treatment reaching similar levels to those found in the normal MCF-10F breast epithelial cells (Fig. 7). Also, the expression of several genes that encode enzymes involved in chromatin modifications such as MGEA5, ATF-2, KDM5B, PRMT2 (PRM2), PHF21A and NSD1, were dysregulated in trMCF clone 11, reaching normal levels after $10^{-6}$ M ATRA treatment (Fig. 7).

\section{Discussion}

In this study we showed that all trans-retinoic acid (ATRA) induced branching of early transformed human breast epithelial cells. The transformed trMCF clone 11 cells form spherical masses in collagen (3D culture) and treatment with $10^{-6} \mathrm{M}$ ATRA produced a significant decrease in spherical masses and an increased number of tubules. Cells at an advanced stage of transformation (bsMCF and caMCF) did not show any change in morphology after being treated with ATRA. Our previous results showed that $\operatorname{RAR} \beta$ (retinoic acid receptor $\beta$ ) was unmethylated in MCF-10F and trMCF cells and became hypermethylated at the invasive (bsMCF) and tumorigenic (caMCF) stages (19); although bsMCF and caMCF were treated with 5-aza-dC to reactivate the expression of RAR $\beta$ in combination with ATRA, no changes in the phenotype of these cells in collagen were observed. Our results indicate that ATRA is able to re-differentiate early transformed cells to a normal stage but, not tumor cells at later stages of the neoplastic process. We previously showed that bsMCF and caMCF had important chromosomal gains and losses and the earlier transformed cells trMCF showed small genomic changes (16); this could explain why ATRA was only effective as a re-differentiation agent in the early transformed breast epithelial cells. Different studies indicate that epigenetic modifications play important roles in RA transcriptional regulation (20-24). Histones have a long N-terminal tail extending outside the nucleosome that is subject to acetylation, phosphorylation, and methylation (25). In the absence of RA, co-repressive elements (SMRT, NCoR and SIN3A) inhibit transcription; the presence of RA releases co-repressors and histone deacetylases 


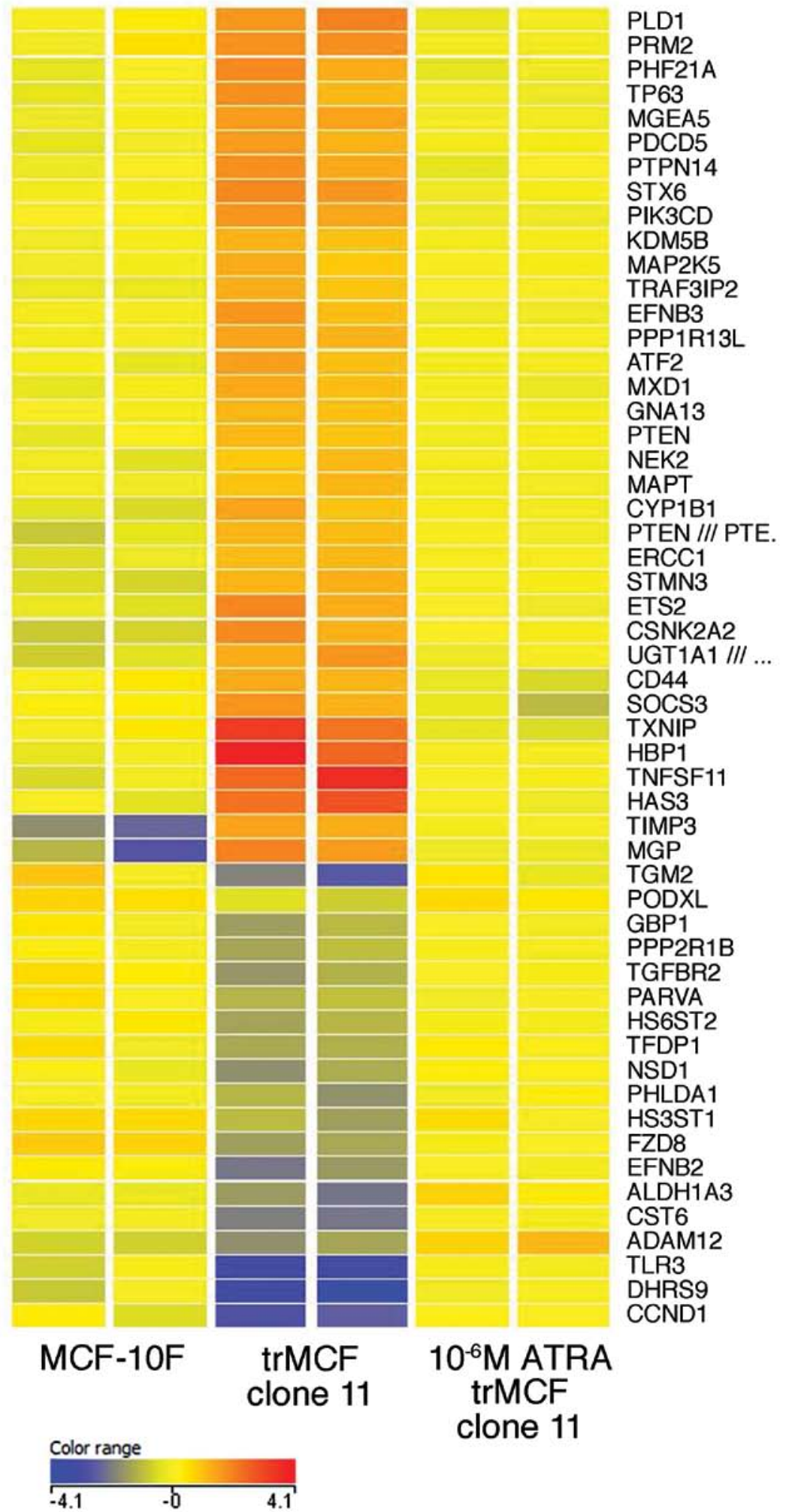

Figure 7. Heat map of selected genes in normal breast epithelial cells and early transformed cells before and after $10^{-6} \mathrm{M}$ ATRA treatment. The expressions of genes involved in cell morphology are shown; also some genes from the aryl hydrocarbon and RAR pathways and genes involved in chromatin modification are shown. The genes that were dysregulated in the early transformed breast epithelial cells (trMCF clone 11) reached normal levels, similar to the normal breast epithelial cells MCF-10F, after treatment with $10^{-6} \mathrm{M}$ ATRA. Red, yellow or blue colors represent expression levels above, at or below the mean level across the samples.

allowing chromatin remodeling and access to specific RAREs $(20,24)$. RA treatment leads to acetylation of histones H3 and $\mathrm{H} 4$ that lead to a more open stage of the chromatin allowing the transcription of ATRA regulated genes. However, only a limited number of information is currently available on the epigenetic dynamics of RA response. 
Recently, analysis of gene expression array datasets of different FDA approved drugs revealed that ATRA (tretinoin) is a drug that is negatively associated with cancer stem cell (CSC) enriched gene expression signature (26). We found that ATRA treatment reduced the expression of the stem cell marker CD44 in early transformed cells. ATRA exerts effects on stem cell differentiation in part via the modulation of the epigenome. Numerous enzymes that alter the modifications on histones are involved in transcriptional activation of specific genes in stem cells, and many of these enzymes are modulated by RA treatment of stem cells (27). The expression of several genes encoding enzymes involved in chromatin modifications such as MGEA5, ATF-2, KDM5B, PRMT2, PHF21A and NSD1 were dysregulated in trMCF clone 11, reaching normal levels after ATRA treatment. Others have shown that in breast cancer, retinoids are effective inhibitors of breast cancer cells at early stages of tumor progression, but their effectiveness diminishes as the tumors become more aggressive (28). Our results support these findings.

Our results show that the RA concentration is important to induced re-differentiation of early transformed breast epithelial cells. The treatment of transformed cells with either $10^{-7}$ or $10^{-8} \mathrm{M}$ ATRA did not induced any change in morphology although, cells were able to form tubules after treatment with $10^{-5}$ and $10^{-6} \mathrm{M}$ ATRA, more tubules being developed after treatment with $10^{-6} \mathrm{M}(1 \mu \mathrm{M})$ ATRA.

Little is known about the genomic targets and effects of the different isoforms of the RARs and mechanism or extent of crosstalk between RA signaling and other signaling pathways. It has been recently shown that RAR binding through the genome is highly coincident with estrogen receptor $\alpha$ binding, resulting in widespread crosstalk of RA and estrogen signaling to antagonistically regulate breastcancer associated genes (29). Our gene expression studies determined 443 genes which defined a signature of ATRA re-programming effect in early transformed breast epithelial cells; these genes were dysregulated in the early transformed cells and they reached normal levels after the cells were treated with $10^{-6} \mathrm{M}$ ATRA. Genes from the aryl hydrocarbon receptor (AhR), retinoic acid receptor (RAR) and the xenobiotic pathways were dysregulated in the early transformed breast epithelial cells and their expression reached normal levels after ATRA treatment. It has been shown that there is an interaction between AhR and RAR activation and that AhR not only binds to polycyclic aromatic hydrocarbon family of environmental contaminants but also to some synthetic retinoids $(30,31)$.

$\mathrm{N}$-(4-hydoxyphenyl) retinamide (fenretinide or 4HPR) is a synthetic retinoid that is currently one of the most promising clinically tested retinoids. The modification of the carboxyl end of all-trans RA with N-4-hydroxyphenyl group resulted in increased efficacy as a chemoprevention agent as well as reduced toxicity when compared with other retinoids (32). Animal models have demonstrated that treatment with fenretinide prevents chemically induced cancers of the breast, prostate, bladder and skin (33-36).

In conclusion, our results showed that $1 \mu \mathrm{M}$ ATRA was able to re-differentiate transformed cells at early stages of the neoplastic process and antagonistically regulated breast cancer associated genes. Our results support previous findings that $1 \mu \mathrm{M}$ ATRA could be used as a chemo-preventive agent to inhibit the progression of premalignant lesions of the breast.

\section{Acknowledgements}

We thank Karen Trush for helping in the preparation of the final figures. This study was supported by The Pennsylvania Breast Cancer Coalition and Friends for an Earlier Breast Cancer Test.

\section{References}

1. Theodosiou M, Laudet V and Schubert M: From carrot to clinic: an overview of the retinoic acid signaling pathway. Cell Mol Life Sci 67: 1423-1445, 2010.

2. Duester G: Retinoic acid synthesis and signaling during early organogenesis. Cell 134: 921-931, 2008.

3. Mongan NP and Gudas LJ: Diverse actions of retinoid receptors in cancer prevention and treatment. Differentiation 75: 853-870, 2007.

4. Connolly R, Nguyen NK and Sukumar S: Molecular pathways: current role and future directions of the retinoic acid pathway in cancer prevention and treatment. Clin Cancer Res 19: 1651-1659, 2013.

5. Bushue $\mathrm{N}$ and Wan YJ: Retinoid pathway and cancer therapeutics. Adv Drug Deliv Rev 62: 1285-1298, 2010.

6. Aagaard MM, Siersbaek R and Mandrup S: Molecular basis for gene-specific transactivation by nuclear receptors. Biochim Biophys Acta 1812: 824-835, 2011.

7. Tsai MJ and O'Malley BW: Molecular mechanisms of action of steroid/thyroid receptor superfamily members. Annu Rev Biochem 63: 451-486, 1994.

8. Rochette-Egly C and Germain P: Dynamic and combinatorial control of gene expression by nuclear retinoic acid receptors (RARs). Nucl Recept Signal 7: e005, 2009.

9. Heyman RA, Mangelsdorf DJ, Dyck JA, et al: 9-cis retinoic acid is a high affinity ligand for the retinoid $\mathrm{X}$ receptor. Cell 68: 397-406, 1992

10. Garattini E, Gianni M and Terao M: Cytodifferentiation by retinoids, a novel therapeutic option in oncology: rational combinations with other therapeutic agents. Vitam Horm 75: 301-354, 2007.

11. Zanardi S, Serrano D, Argusti A, Barile M, Puntoni M and Decensi A: Clinical trials with retinoids for breast cancer chemoprevention. Endocr Relat Cancer 13: 51-68, 2006.

12. Seewaldt VL, Johnson BS, Parker MB, Collins SJ and Swisshelm K: Expression of retinoic acid receptor beta mediates retinoic acid-induced growth arrest and apoptosis in breast cancer cells. Cell Growth Differ 6: 1077-1088, 1995.

13. Swisshelm K, Ryan K, Lee X, Tsou HC, Peacocke M and Sager R: Down-regulation of retinoic acid receptor beta in mammary carcinoma cell lines and its up-regulation in senescing normal mammary epithelial cells. Cell Growth Differ 5: 133-141, 1994.

14. Widschwendter M, Berger J, Muller HM, Zeimet AG and Marth C: Epigenetic downregulation of the retinoic acid receptor-beta2 gene in breast cancer. J Mammary Gland Biol Neoplasia 6: 193-201, 2001.

15. Russo J, Fernandez SV, Russo PA, et al: 17-Beta-estradiol induces transformation and tumorigenesis in human breast epithelial cells. FASEB J 20: 1622-1634, 2006.

16. Huang Y, Fernandez SV, Goodwin S, et al: Epithelial to mesenchymal transition in human breast epithelial cells transformed by 17beta-estradiol. Cancer Res 67: 11147-11157, 2007.

17. Fernandez SV and Russo J: Estrogen and xenoestrogens in breast cancer. Toxicol Pathol 38: 110-122, 2010.

18. Hebner C, Weaver VM and Debnath J: Modeling morphogenesis and oncogenesis in three-dimensional breast epithelial cultures. Annu Rev Pathol 3: 313-339, 2008.

19. Snider KE, Ehya H, Russo J and Fernandez SV: NRG1 and RARB hypermethylation in breast cancer progression. Proceedings of the ACCR 102nd Anual Meeting, Orlando, FL. Cancer Res 71 (Suppl 1): abs. 75, 2011.

20. Glass CK and Rosenfeld MG: The coregulator exchange in transcriptional functions of nuclear receptors. Genes Dev 14: 121-141, 2000. 
21. Hartman HB, Yu J, Alenghat T, Ishizuka T and Lazar MA: The histone-binding code of nuclear receptor co-repressors matches the substrate specificity of histone deacetylase 3. EMBO Rep 6: 445-451, 2005.

22. Lefebvre B, Ozato K and Lefebvre P: Phosphorylation of histone $\mathrm{H} 3$ is functionally linked to retinoic acid receptor beta promoter activation. EMBO Rep 3: 335-340, 2002.

23. McKenna NJ and O'Malley BW: Combinatorial control of gene expression by nuclear receptors and coregulators. Cell 108: 465-474, 2002.

24. Rochette-Egly C: Dynamic combinatorial networks in nuclear receptor-mediated transcription. J Biol Chem 280: 32565-32568, 2005.

25. Chen H, Lin RJ, Xie W, Wilpitz D and Evans RM: Regulation of hormone-induced histone hyperacetylation and gene activation via acetylation of an acetylase. Cell 98: 675-686, 1999.

26. Bhat-Nakshatri P, Goswami CP, Badve S, Sledge GW Jr and Nakshatri H: Identification of FDA-approved drugs targeting breast cancer stem cells along with biomarkers of sensitivity. Sci Rep 3: 2530, 2013.

27. Gudas LJ: Retinoids induce stem cell differentiation via epigenetic changes. Semin Cell Dev Biol 24: 701-705, 2013.

28. Zhang XK, Liu Y and Lee MO: Retinoid receptors in human lung cancer and breast cancer. Mutat Res 350: 267-277, 1996.

29. Hua S, Kittler R and White KP: Genomic antagonism between retinoic acid and estrogen signaling in breast cancer. Cell 137: 1259-1271, 2009.
30. Soprano DR and Soprano KJ: Pharmacological doses of some synthetic retinoids can modulate both the aryl hydrocarbon receptor and retinoid receptor pathways. J Nutr 133: 277S-281S, 2003.

31. Murphy KA, Quadro L and White LA: The intersection between the aryl hydrocarbon receptor (AhR)- and retinoic acid-signaling pathways. Vitam Horm 75: 33-67, 2007.

32. Hail N Jr, Kim HJ and Lotan R: Mechanisms of fenretinideinduced apoptosis. Apoptosis 11: 1677-1694, 2006.

33. McCormick DL, Becci PJ and Moon RC: Inhibition of mammary and urinary bladder carcinogenesis by a retinoid and a maleic anhydride-divinyl ether copolymer (MVE-2). Carcinogenesis 3: 1473-1476, 1982.

34. McCormick DL and Moon RC: Antipromotional activity of dietary N-(4-hydroxyphenyl)retinamide in two-stage skin tumorigenesis in CD-1 and SENCAR mice. Cancer Lett 31: 133-138, 1986.

35. Moon RC, Pritchard JF, Mehta RG, Nomides CT, Thomas CF and Dinger NM: Suppression of rat mammary cancer development by $\mathrm{N}$-(4-hydroxyphenyl)retinamide (4-HPR) following surgical removal of first palpable tumor. Carcinogenesis 10: 1645-1649, 1989.

36. Pollard M, Luckert PH and Sporn MB: Prevention of primary prostate cancer in Lobund-Wistar rats by $\mathrm{N}$-(4-hydroxyphenyl) retinamide. Cancer Res 51: 3610-3611, 1991. 